\section{CHIAPAS 2019}

XV Congreso Latinoamericano de Patología de Construcción y

XVII Congreso de Control de Calidad en la Construcción

CONPAT 2019, Vol. 1, CONTROL DE CALIDAD DOI: https://doi.org/10.21041/CONPAT2019/V3REC385CMP CONFERENCIA MAGISTRAL PLENARIA Tuxtla Gutiérrez, Chiapas, México Editado por: Francisco Alberto Alonso Farrera,

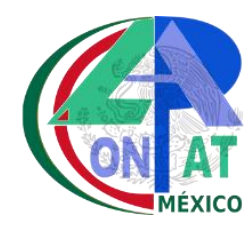
Francisco Javier Olguín Coca

\title{
INCIDENCIA DEL ÁRIDO RECICLADO SOBRE LA DURABILIDAD DEL HORMIGÓN ARMADO FRENTE A LA CORROSIÓN
}

\author{
Y. A. VILLAGRÁN-ZACCARDI, C. M. PICO-CORTÉS, C. J. ZEG \\ ${ }^{1}$ LEMIT, CONICET, La Plata, Argentina.
}

\begin{abstract}
RESUMEN
El árido reciclado procedente de hormigón de desecho triturado es apto para elaborar hormigón estructural. Permite consumir menos recursos no renovables y generalmente también menos energía. Pero es más poroso que la mayoría de las rocas naturales. La porosidad del hormigón de recubrimiento define la resistencia frente a la corrosión de las armaduras, y entonces se puede dudar acerca de cómo el empleo del árido reciclado afecta a la durabilidad del hormigón armado. En este artículo se describe el desempeño frente a la corrosión de hormigones con áridos reciclados, en las etapas de iniciación (carbonatación e ingreso de cloruro) y propagación (resistividad eléctrica y fisuración). Se enuncian aspectos particulares a considerar, mientras se verifica la factibilidad de incluir árido reciclado en hormigón sin disminuir sus prestaciones durables.
\end{abstract}

Palabras clave: hormigón con árido reciclado; corrosión; carbonatación; ingreso de cloruro; durabilidad. 


\section{INTRODUCCIÓN}

El uso de áridos reciclados a partir de hormigón triturado permite disminuir el impacto ambiental de los residuos de construcción y demolición, reduciendo la cantidad de vertederos y la extracción de rocas. En virtud de prácticas eco-eficientes, se ha aplicado durante varias décadas, principalmente en los Estados Unidos, Japón y Europa, y cada vez más en América Latina. Después de la Segunda Guerra Mundial, se despertó un gran interés en el reciclaje de residuos de hormigón debido a la urgente necesidad de reconstruir la infraestructura en los países afectados. A finales de la década de 1970, 40 millones de toneladas de hormigón residual se habían reutilizado en la antigua Unión Soviética (Xiao, 2018). Las regulaciones japonesas para el uso de hormigón reciclado datan de 1977, lo que permitió que para 1988 la proporción de reciclaje de desechos de hormigón en Tokio fuera tan alta como 56\% (Xiao, 2018). En Alemania, el uso de áridos reciclados se enmarca en las especificaciones para agregados, en general, desde 1998. Los programas intensivos de modernización de infraestructura produjeron un volumen significativo de instalaciones construidas con hormigón con árido reciclado desde principios de 1990 (Xiao, 2018). En países con escasez de áridos naturales como Países Bajos o Bélgica, las tasas de reciclado superan el $80 \%$, aunque sólo una fracción de estos áridos son usados para fabricar nuevo hormigón. Por lo tanto, es necesario conocer con fiabilidad las propiedades del hormigón con árido reciclado.

Los áridos reciclados tienen características diferentes a los áridos gruesos naturales debido al mortero adherido a sus partículas (Hansen, 1986; Xiao, 2018; ACI Committee 555, 2001). Así que, mientras que las propiedades del árido natural dependen principalmente de las características de la roca original, las características del árido reciclado están relacionadas no solo con las de la roca sino también con las del hormigón de origen (Rasheeduzzafar y Khan, 1984; Zega et al., 2010). Existe un acuerdo general acerca de que los áridos reciclados generalmente tienen propiedades menos convenientes que los áridos naturales, es decir, menor densidad, menores resistencias a la acción de las heladas y a la abrasión, mayor material que pasa el tamiz a $75 \mu \mathrm{m}$ y mayor absorción. Como resultado, las proporciones de la mezcla y las propiedades frescas y endurecidas de los hormigones con áridos reciclados son diferentes de las de los áridos naturales convencionales (Limbachiya et al., 2000; Zega y Di Maio, 2003; Topçu y Şengel, 2004).

La influencia del árido grueso reciclado en las propiedades mecánicas del hormigón ha sido ampliamente estudiada. En general, se concluye que los índices de reemplazo de hasta el 30\% de árido grueso natural por árido grueso reciclado no producen cambios significativos en las propiedades mecánicas (Limbachiya et al., 2000; Gómez et al., 2001; Padmini et al., 2009). Además, algunos estudios (Di Maio et al., 2005; Zega y Di Maio, 2007) indican que incluso proporciones de reemplazo de hasta el $75 \%$ producen influencias insignificantes en las propiedades mecánicas de los hormigones. Con respecto al desempeño durable de los hormigones con árido reciclado, existe cierto desacuerdo en la literatura y se pueden encontrar resultados contradictorios con respecto a la medida en que este árido puede influir en las propiedades durables del hormigón. Esto probablemente se deba a los efectos opuestos en la estructura de los poros, por una parte, se encuentra la mejora de la zona de transición de interfase entre el árido reciclado y el nuevo mortero (Otsuki et al., 2003), y por otra parte, la mayor porosidad de estos áridos, que aumenta la porosidad total de hormigón. Luego, la durabilidad del hormigón con áridos reciclados se vinculará con el efecto del árido en los parámetros de transporte relacionados, como el ingreso de cloruro, el transporte de agua y humedad y la resistencia a ataques físico-químicos, como el ataque de sulfato o el congelamiento y deshielo. Para cada caso, el predominio de una zona de interfase mejorada o una porosidad más alta que en el hormigón con árido natural explicará una durabilidad mejorada o disminuida del hormigón con árido reciclado.

Las propiedades de transporte del hormigón de recubrimiento son de fundamental importancia en el estudio de la durabilidad del hormigón armado. El flujo de materia se produce principalmente por permeabilidad (motivada por un gradiente de presiones), difusividad (debido a un gradiente de 
concentraciones), y absorción capilar (causada por succión capilar). Para evitar la corrosión de las armaduras, en estructuras en ambientes marinos debe procurarse aminorar el ingreso de cloruro, y en otros ambientes aminorar la velocidad de carbonatación. En principio, el acero empotrado en el hormigón se encuentra en estado pasivo, pero la caída del pH debida a la carbonatación o la presencia de cloruro en cantidad suficiente producen la rotura de la capa pasiva (Tuutti, 1982; Huerta, 1997). Además, para que se produzca corrosión a una velocidad considerable, es necesaria la presencia simultánea de una alta humedad relativa y oxígeno en el medio. Por lo tanto, el efecto del árido reciclado sobre la durabilidad respecto a la corrosión de armaduras está fuertemente vinculado con la forma en que afecta las propiedades de transporte.

\section{POROSIDAD Y PROPIEDADES DE TRANSPORTE DE HUMEDAD}

El árido reciclado tiene mayor capacidad de absorción en comparación con los áridos de origen natural usados habitualmente (Zega y Di Maio, 2007), por lo que incrementa la porosidad del hormigón y su incorporación puede resultar perjudicial respecto a las propiedades de durabilidad de las armaduras (Rasheeduzzafar y Khan, 1984). Sin embargo, en ciertos casos la afectación puede ser muy pequeña (Tanaka et al., 2004; Villagrán et al., 2005).

Si bien las propiedades de transporte del hormigón están relacionadas con el volumen de poros del material, aún más importantes son la distribución y la conectividad de los mismos (Fernández Luco, 2001), siendo los poros conectados con el exterior los que permiten el acceso de sustancias perjudiciales. Por lo tanto, el aumento de la porosidad del hormigón no es suficiente para juzgar el efecto del árido reciclado sobre la durabilidad.

La porosidad del árido no es importante solamente en el hormigón endurecido, sino también durante su elaboración. La absorción latente por parte de los áridos juega un rol principal al ser capaz de modificar el contenido de agua libre en la mezcla y por lo tanto disminuir su trabajabilidad. En el caso de los áridos gruesos reciclados esta situación es relativamente sencilla de resolver. Estos áridos pueden ser previamente saturados en agua, o puede adicionarse el agua de absorción de los áridos a la mezcla y permitir el tiempo necesario para que los mismos absorban. En estos casos, se asegura que la relación agua/cemento efectiva y contenido de agua libre no se vean afectados. En el caso de los áridos finos reciclados el problema es un poco más complejo. Por una parte, la forma de las partículas origina por sí misma una mayor demanda de agua para un cierto asentamiento, con el fin de lubricar y disminuir la fricción interna del esqueleto granular. Por otra parte, los métodos usualmente utilizados para determinar la capacidad de absorción de estos áridos finos no son siempre efectivos. Eventualmente, se acaba modificando la cantidad de agua libre sin intención. De esta forma, los áridos pueden afectar las propiedades durables de una estructura en forma indirecta al disminuir su compactabilidad.

Actualmente, los métodos de determinación de la capacidad de absorción de los áridos finos son confiables sólo para el caso de materiales con partículas redondeadas (arenas naturales). En cambio, métodos para determinar la condición saturada a superficie seca, como el del cono truncado, resultan en errores significativos cuando son aplicados a áridos de trituración como el árido fino reciclado (Sosa et al., 2018a). Frente a esta incertidumbre, parece inconveniente corregir el contenido de agua de la mezcla para la absorción total de agua del árido fino reciclado (Sosa et al., 2018b). Ya que los áridos efectivamente absorben cierta cantidad de agua, parece más apropiado utilizar una fracción de la capacidad de absorción de agua del árido, y a partir del análisis de la literatura, utilizar un valor equivalente al $70 \%$ de la absorción total determinada mediante el método del cono truncado. Efectivamente, este coeficiente depende del método de ensayo para la determinación de la absorción de agua del árido fino. De esta forma, mucha literatura que analiza y concluye un efecto muy desfavorable del árido fino reciclado sobre las propiedades durables, no resulta del todo convincente en cuanto la relación agua/cemento efectiva pudo haberse visto incrementada al adicionar toda el agua de 
absorción estimada, haciendo estas mezclas incomparables con las del hormigón convencional con áridos de mucha menor absorción y por lo tanto, menor relación agua/cemento efectiva.

En (Sosa et al., 2018b) se determinó que las propiedades mecánicas y durables del hormigón con árido fino reciclado están mejor correlacionadas con la relación agua/cemento total que con la relación agua/cemento efectiva estimada a partir de la absorción del árido. Ello es debido a que, en la literatura, las correcciones de agua en la mezcla debidas a la absorción del árido fino reciclado tienden a aumentar la relación agua/cemento efectiva involuntariamente. En consecuencia, está claro que los resultados obtenidos para la durabilidad y las propiedades de transporte de esos hormigones con árido fino reciclado son lógicamente menos favorables que las del hormigón convencional en el que no se ha realizado ninguna corrección o una corrección mucho menor del agua de mezclado.

En otros estudios de durabilidad (Zega et al., 2016; Zega y Di Maio, 2011), se ha demostrado que las partículas de árido poroso pierden significancia cuando son incorporadas en una matriz poco porosa. Los áridos reciclados permiten diseñar hormigones durables en tanto y en cuanto se alcance el desempeño requerido para el hormigón que constituye, y estudios experimentales han demostrado que esto es ampliamente factible.

Un aspecto a considerar de los estudios de laboratorio que comparan el hormigón reciclado con el convencional es que involucran, en general, a muestras ampliamente curadas, es decir con un alto grado de hidratación. Esto desconsidera un beneficio potencial del árido reciclado, que es más poroso y podría proporcionar agua de curado si no se aplica un curado externo (Kovler y Jensen, 2007; Kim y Bentz, 2008; Zega et al., 2014).

\section{CARBONATACIÓN}

La resistencia a la carbonatación y otras propiedades durables del hormigón con árido reciclado se han estudiado por distintos autores (Werle et al., 2011; Silva et al., 2015; Leemann y Loser, 2019). El efecto del árido en el desempeño durable se ha relacionado principalmente con el aumento causado en la porosidad total de la mezcla, pero aún deben abordarse varios aspectos. La carbonatación ocurre cuando el dióxido de carbono penetra en el hormigón y reduce su alcalinidad al fijar y precipitar el calcio y otros álcalis en forma de carbonatos. El árido reciclado puede afectar a la carbonatación en dos aspectos importantes. Por un lado, el mortero adherido en las partículas del árido aumenta la porosidad de la mezcla y permite una mayor velocidad de transporte del dióxido de carbono. Por esta razón, a veces se recomienda una reducción en la relación agua/cemento cuando se usan áridos reciclados como reemplazo parcial de áridos naturales (Silva et al., 2015; Faleschini et al., 2018). También se ha recomendado limitar el porcentaje de reemplazo (por ejemplo, a un máximo del 30\%) para evitar efectos significativos del incremento de la porosidad por el mortero adherido en la velocidad de carbonatación (Xiao et al., 2010). Por otro lado, el mortero adherido puede proporcionar una cierta cantidad de productos de hidratación no carbonatados que podrían contribuir a la resistencia a la carbonatación al aumentar la cantidad de dióxido de carbono que la mezcla de hormigón puede fijar por unidad de volumen. A pesar del avance en el abordaje de esta temática, la resistencia a la carbonatación del hormigón con árido reciclado no se ha resuelto todavía.

El proceso de carbonatación adquiere interés de ingeniería no sólo porque permite la corrosión del refuerzo, sino que también porque produce cambios en la microestructura del hormigón. La reducción de la alcalinidad que se produce después de la carbonatación provoca la despasivación de las barras de acero empotradas que se ven protegidas por el alto $\mathrm{pH}$. Sin embargo, algunos cambios microestructurales en la estructura de poros del hormigón carbonatado pueden considerarse positivos. Cuando se utiliza cemento Portland normal, tras la precipitación de carbonatos en los poros, la conectividad de los poros se reduce y se puede afectar notablemente a las propiedades de transporte del hormigón. Este aspecto particular debe observarse para el caso del hormigón con árido reciclado, ya que el mortero adherido no carbonatado también puede beneficiarse de este proceso, y el impacto 
relativo de la carbonatación en el hormigón con árido reciclado puede ser mayor que para una mezcla de hormigón convencional de proporciones comparables. En el hormigón con alto contenidos de materiales cementíceos suplementarios el tema es algo diferente. El agotamiento de la portlandita causado por el consumo concurrente de la reacción puzolánica y la carbonatación deteriora la estructura del silicato de calcio hidratado al descalcificarla, debilitando la microestructura del hormigón. Por lo tanto, el problema de la carbonatación no se reduce al estudio de la porosidad.

Para la predicción de la velocidad de carbonatación, generalmente se consideran las propiedades mecánicas a 28 días y se aplican relaciones empíricas. De hecho, el diseño basado en prestaciones para la durabilidad generalmente considera propiedades de este tipo para estipular los requisitos para la mezcla de hormigón. En (Zega et al., 2019) se determinó la correlación entre los coeficientes de carbonatación de hormigones reciclados y las propiedades a 28 días. El coeficiente de absorción capilar como un índice de predicción para la carbonatación es generalmente considerada por las guías para durabilidad como una herramienta idónea de diseño, y el uso de árido reciclado se asocia a esta relación. Por lo tanto, un bajo coeficiente de absorción capilar es indicativo de un hormigón poco carbonatable, aún si se utilizan áridos reciclados en la mezcla. A la inversa, la resistencia a la compresión parece ser un descriptor muy débil del efecto del árido reciclado en el desempeño del hormigón frente a la carbonatación. Ello demuestra que la afectación de la velocidad de carbonatación no viene dada únicamente por el volumen de poro. Precisamente, estas conclusiones fueron indicadas en (Silva et al., 2015), quienes relacionaron la influencia del árido reciclado sobre la resistencia a la carbonatación con el volumen de poro que el árido reciclado proporciona a la mezcla. Sin embargo, los resultados en (Zega et al., 2019) sugieren que, bajo exposición natural, se debe poner atención a un cierto rango de tamaños de poros. En relación a la carbonatación, un hormigón reciclado puede cumplir apropiadamente los requerimientos habitualmente establecidos para el hormigón convencional.

\section{INGRESO DE CLORURO}

La durabilidad del hormigón armado en ambiente marino resulta dependiente de la resistencia del hormigón de recubrimiento al ingreso de cloruro. El ingreso de cloruro en el hormigón conduce a la despasivación localizada de armaduras. Cuando se alcanza un contenido suficiente de cloruro, contenido umbral, se produce la rotura localizada de la capa pasiva. Entonces, la entrada de cloruro en el hormigón define la duración del período de iniciación para la corrosión del acero de refuerzo. Sin embargo, parte del cloruro que ingresa en el hormigón puede ser retenido en la matriz de cemento y participar en el proceso de despasivación. Esto es de gran importancia, ya que solo los cloruros libres pueden continuar penetrando en el hormigón y es el cloruro libre el que potencialmente causa la ruptura de la capa pasiva. El ingreso de cloruro en hormigón es un tema de amplia importancia para la durabilidad del hormigón armado en ambiente marino y por lo tanto debe ser controlado.

La resistencia del hormigón a la penetración del cloruro se logra principalmente reduciendo la porosidad del material (Collepardi et al., 1972; Monosi et al., 1989). Esta porosidad se define en gran medida por la relación agua/material cementíceo $(\mathrm{a} / \mathrm{mc})$, grado de hidratación, contenido de cemento, grado de compactación y proporción de árido grueso/fino. Por otro lado, la capacidad de retención de cloruro de la matriz es una función no lineal del contenido total de cloruro, y está en relación con la cantidad de productos de hidratación (Tang y Nilsson, 1993). Por lo tanto, el diseño de la mezcla es la herramienta clave para lograr estructuras de hormigón armado durables.

Con respecto a la influencia del árido reciclado en la penetración del cloruro, se pueden encontrar diferentes resultados en la literatura. Mientras que algunos autores concluyen que el uso de árido grueso reciclado aumenta la velocidad de penetración del cloruro (Rasheeduzzafar y Khan, 1984), otros informan que las diferencias entre los hormigones convencionales y los reciclados son insignificantes (Tanaka et al., 2004; Villagrán-Zaccardi et al., 2008), incluso cuando el 100\% del árido grueso se constituye por árido reciclado (Limbachiya et al., 2000; Otsuki et al., 2003). 
La mayoría de las investigaciones al respecto consisten en exponer al hormigón sumergiéndolo en una solución de $\mathrm{NaCl}$ (generalmente de concentración de $30 \mathrm{~g} / \mathrm{l}$ ). Aunque estas pruebas de laboratorio aceleradas son útiles para la caracterización cuantitativa de la resistencia al ingreso de cloruro, no son completamente representativas de la exposición atmosférica marina, donde las variaciones debidas a eventos de lluvia, viento y la concentración superficial variable de cloruro en el tiempo son condiciones influyentes. Además, la presencia de sulfato en el agua de mar puede reducir la capacidad de retención de cloruro en comparación con la exposición a una solución de $\mathrm{NaCl}$ pura (Villagran y Matiasich, 2004). Por lo tanto, es importante evaluar la resistencia a la penetración de cloruros mediante pruebas de laboratorio aceleradas, pero también complementarlas con estudios de larga duración en exposición marina.

En un estudio anterior (Villagrán-Zaccardi et al., 2008), se abordó la influencia relativa de diversas variables sobre la penetración de cloruro en el medio marino y en solución de $\mathrm{NaCl}$, comparando hormigón con árido grueso reciclado y hormigón convencional. Los resultados de este estudio indican que la porosidad del árido reciclado adquiere importancia para relaciones agua cemento menores a 0,35 , donde los frentes de concentración fueron hasta dos veces más profundos que los del hormigón convencional. Pero para relaciones agua/cemento iguales o superiores a 0,40 , el árido reciclado no mostró influencia detectable. Mientras que la velocidad de penetración de cloruro fue más alta en el hormigón reciclado, el mismo también demostró una capacidad adicional de retención de cloruro debido al mortero adherido. Por lo tanto, existe una compensación de la porosidad por parte del contenido de productos de hidratación.

En una investigación relacionada (Zega et al., 2015), se demostró que el tipo de árido natural que constituye al hormigón a ser reciclado tiene una significancia importante. La petrografía y textura del árido genera diferencias significativas en la zona de interfase. En coincidencia con los resultados en (Villagrán-Zaccardi et al., 2008), para un contenido de árido reciclado del 25\% se obtuvieron perfiles de ingreso de cloruro similares en hormigón convencional y reciclado. Cuando el porcentaje de reemplazo se incrementó al $75 \%$, en los casos en que se reemplazó árido de textura lisa por árido reciclado, se notó incluso cierta mejora atribuida a mejoras en la zona de interfase. En (Zega et al., 2015), también se observó una mayor capacidad de retención de cloruro del hormigón reciclado respecto al convencional, asociada a un mayor contenido de productos de hidratación.

La matriz cementícea posee la capacidad de inmovilizar cierta proporción del cloruro que ingresa. Esto es importante ya que el cloruro libre es el que resulta perjudicial respecto a la durabilidad de las armaduras de acero dispuestas en el interior del hormigón. La capacidad de retención de cloruro presenta una dependencia no lineal de la concentración total de cloruro, influenciada principalmente por el grado de hidratación del cemento (Tang y Nilsson, 1993) y formación tardía de productos de hidratación (Delagrave et al., 1997). Los efectos de la retención de cloruro conducen a un retardo en el ingreso de cloruro en el hormigón. En una sección transversal, una fracción del total de cloruro ingresante quedará inmovilizada en la matriz y el resto permanecerá libre para continuar penetrando. En efecto, la retención de cloruro en el hormigón reduce la cantidad de cloruro libre, y su influencia sobre el inicio de la corrosión es doble (Nilsson, 1996): 1) la velocidad del transporte iónico en el hormigón se reduce; y 2) la cantidad de cloruro libre que se acumula en la superficie del acero de refuerzo y propicia el picado es menor. Por lo tanto, los modelos de ingreso de cloruro son significativamente dependientes de la relación de retención asumida entre cloruro libre en la solución de poro del hormigón y el cloruro retenido en los productos de hidratación (Martín-Pérez, 1999). La relación entre cloruro fijo y libre no es permanente, ya que además de depender del contenido de cloruro fijado, también influyen el pH del medio (Glass et al., 2000) (más exactamente la cantidad de iones hidróxido libres), la temperatura, la humedad relativa y la presencia de otros aniones.

Por lo tanto, los áridos reciclados tienen la capacidad potencial de incrementar la capacidad de retención de cloruro (Villagrán-Zaccardi et al., 2008; Zega et al., 2015), debido principalmente al contenido de cemento correspondiente al mortero adherido en el árido reciclado. Esta capacidad potencial puede 
eventualmente contrarrestar inicialmente efectos negativos de una mayor porosidad del tipo de árido sobre la velocidad de ingreso de cloruro. Al mismo tiempo, el contenido de mortero adherido y sus propiedades dependen en gran medida de las características del hormigón original del cual provienen (Zega et al., 2010), con lo cual es esperable que el incremento en la capacidad de cloruro debido al árido reciclado sea variable.

\section{RESISTIVIDAD}

La resistencia eléctrica se define como la relación entre el voltaje aplicado y la corriente eléctrica que fluye a través de una muestra. También se puede definir como la resistencia de los materiales al paso de corriente eléctrica. Esta propiedad se define por unidad de área y longitud, denominándose como resistividad. Es importante conocer la resistencia del hormigón al flujo de corriente eléctrica porque contribuye a determinar el riesgo de corrosión del acero empotrado. La resistividad convencional del hormigón permite clasificarlo en cuatro tipos generales: <100, 100-500, 500-1000 y >1000 $\Omega$ m (COST 509, 1997), para los cuales el riesgo de corrosión del refuerzo es alto, moderado, bajo y despreciable, respectivamente. Una consideración importante es diferenciar a la resistividad en masa de la resistividad superficial (método Wenner), ya que los valores entre ambos difieren y no resultan directamente comparables (Azarsa y Gupta, 2017).

La resistividad del hormigón en estado saturado es una función inversa de la porosidad del material y la conductividad del líquido de poro. Ya que el uso de árido reciclado no cambia notablemente a la conductividad del líquido de poro del hormigón, resulta lógico que un árido con mayor porosidad como lo es el reciclado disminuya linealmente la resistividad del hormigón (Arredondo-Rea et al., 2019).

Sin embargo, la interpretación directa de que una menor resistividad en estado saturado implica una mayor probabilidad de corrosión no es exacta. El hormigón que no está en contacto directo con agua desarrolla un equilibrio higroscópico con el medio circundante, a partir de la condensación en su porosidad interna. De esta forma resulta necesaria la comprobación de la resistividad en estado no saturado para la determinación de probabilidad de corrosión en la mayoría de las estructuras. Esta medición no es sencilla y requiere técnicas particulares (Villagran Zaccardi y Di Maio, 2014).

Al incorporar áridos reciclados, la reducción en la resistividad eléctrica indica un efecto en la interconexión de poros en el hormigón. El factor de formación relaciona a la resistividad del hormigón con la resistividad del líquido de poro según la ley de Archie (Whittington et al., 1981). El factor de formación de los hormigones aumenta con el contenido de árido reciclado, pero el factor de formación de la fase pasta se incrementa ligeramente (Omary et al., 2018). Esto significa que, si bien el mortero adherido incrementa la conectividad de poros, el efecto dinámico de absorción del árido puede mejorar la zona de interfase notablemente.

\section{FISURACIÓN POR CORROSIÓN}

Respecto a la despasivación por cloruro, en (Villagrán Zaccardi et al., 2009) se demostró que la despasivación ocurre indistintamente respecto a la inclusión de áridos gruesos reciclados, y depende del contenido umbral de cloruro del mismo modo que en el hormigón convencional.

El uso de árido reciclado introduce más interfaces en el hormigón, lo que acelera la propagación de fisuras inducidas por la corrosión en el hormigón de recubrimiento (Zhao et al., 2014). Sin embargo, la corrosión del acero y el proceso de fisuración inducido por la corrosión en el hormigón no se ven influenciados significativamente al utilizar una pequeña cantidad de árido grueso reciclado, por ejemplo $33 \%$ del volumen total de árido grueso (Zhao et al., 2014). Esto puede deberse a que la disminución del módulo de elasticidad del hormigón también mejora su compatibilidad mecánica y las tensiones desarrolladas por los productos de formación se disipan más fácilmente. La porosidad interna del 
hormigón dispone espacios para la precipitación de los productos de corrosión, y por lo tanto se generan menores tensiones internas cuanto mayor es la porosidad del hormigón. Por lo tanto, parece existir un contenido óptimo de árido reciclado que favorece la disipación de tensiones generados por los productos de corrosión.

Por otra parte, (Ortega et al., 2010) determinaron la disminución de la primera frecuencia natural, de vigas elaboradas con árido reciclado, sometidas a corrosión acelerada y bajo carga flexional. El daño y caída en la rigidez de estas vigas fue más acentuado al comienzo de la fisuración que al final de los ensayos. El crecimiento de la fisuración se desacelera con el avance de la penetración de la corrosión, pero la profundidad media del ataque a las armaduras se acelera al mismo tiempo. La pérdida rápida de adherencia entre acero y hormigón reciclado se debe principalmente a la mayor conductividad eléctrica debida a su mayor porosidad. Sin embargo, no se manifestaron indicios de un desarrollo diferente de la fisuración en comparación con el hormigón convencional. En este sentido, al contrario de lo que podría pensarse, la mayor porosidad del material enmascara el deterioro al retrasar la aparición de fisuración externa para niveles avanzados de corrosión.

El menor desarrollo de fisuración para igual nivel de corrosión fue observado en (Moro et al., 2016) para hormigones con entre 0 y $100 \%$ de árido grueso reciclado. Los mayores anchos de fisuras y el crecimiento más acelerado de los mismos fueron observados para las vigas con hormigón convencional, disminuyendo a medida que aumenta el porcentaje de reemplazo de árido natural por reciclado. De esta forma, el desarrollo de fisuración por corrosión en estructuras con árido reciclado no necesariamente se verá afectado desfavorablemente por la mayor porosidad del hormigón.

\section{CONCLUSIONES}

La incidencia del árido reciclado sobre la durabilidad del hormigón armado frente a la corrosión ha sido investigada ampliamente, pero el tema aún no ha sido totalmente dilucidado.

El incremento de la porosidad del hormigón de recubrimiento en general tiende a definir que el árido reciclado es perjudicial por ser más poroso que la mayoría de los áridos naturales, pero existen algunos aspectos adicionales que deben ser considerados.

En este artículo se analizó información en la literatura respecto al desempeño frente a la corrosión de hormigones con áridos reciclados, tanto en las etapas de iniciación (carbonatación e ingreso de cloruro) y propagación (resistividad eléctrica y fisuración). Los aspectos particulares a considerar en cada caso son:

- El aumento de la porosidad del hormigón no es suficiente para juzgar el efecto del árido reciclado sobre la durabilidad, ya que también deben evaluarse la conectividad de poro. Si bien el mortero adherido produce un aumento general de la conectividad de poro del hormigón, el efecto del proceso dinámico de absorción de las partículas parece producir una mejora en la zona de interfase. Este aspecto es sugerido por resultados de resistividad de hormigones con áridos reciclados y el factor de formación asociado. Resulta conveniente investigar este aspecto con mayor profundidad.

- El mortero adherido en el árido reciclado puede proporcionar una reserva adicional de productos de hidratación carbonatables, y por lo tanto aumentar la capacidad de retención de dióxido de carbono del hormigón. La carbonatación también produce cambios en la microestructura del hormigón, y, por lo tanto, un tratamiento de carbonatación puede ser adecuado para reducir la porosidad del árido antes de su utilización en hormigón nuevo.

- El ingreso de cloruro en hormigón con árido reciclado es más rápido cuando se encuentra saturado, en función de la porosidad total del hormigón. Sin embargo, en hormigón parcialmente saturado la situación no es tan clara. Ya que el transporte de cloruro se produce en la fase de poros conteniendo líquido y la diferencia en la mesoporosidad debida al árido reciclado no es tan significativa, la velocidad de ingreso de cloruro en hormigón no saturado no se incrementa notablemente, a menos 
que se trate de hormigón con muy baja relación agua/cemento. Por otra parte, el contenido de productos de hidratación del mortero adherido provee una capacidad de retención de cloruro adicional y facilita el retraso del proceso de ingreso.

- La conductividad de hormigón saturado aumenta con el contenido de árido reciclado en función del aumento de la porosidad total. Sin embargo, en hormigón no saturado el incremento relativo es menos significativo debido al contenido similar de líquido de poro en el hormigón con árido reciclado que en el hormigón convencional. Por otra parte, el factor de formación de la matriz generada en hormigones incorporando áridos reciclados resulta relativamente mejorado respecto a hormigones con áridos no porosos. Esta incidencia sugiere una mejora en la zona de interfase o la conectividad de la matriz misma y por lo tanto merece mayores investigaciones.

- El desarrollo de fisuración por corrosión está relacionado con el mayor número de interfases en el hormigón con árido reciclado. Sin embargo, la mayor porosidad del material puede conducir a enmascarar niveles significativos de deterioro sin sintomatología externa significativa. En este sentido deben realizarse mayores investigaciones respecto a la capacidad portante por pérdida de adherencia debida a la corrosión en elementos con hormigón reciclado.

\section{AGRADECIMIENTOS}

Los autores agradecen el apoyo financiero de FONCyT a través del PICT 2017-0091 para la realización de esta publicación.

\section{REFERENCIAS}

ACI Committee 555 (2001). Removal and Reuse of Hardened Concrete - ACI 555-01.

Arredondo-Rea, S.P., Corral-Higuera, R., Gómez-Soberón, J.M., Gámez-García, D.C., BernalCamacho, J.M., Rosas-Casarez, C.A., and Ungsson-Nieblas, M.J. (2019). Durability Parameters of Reinforced Recycled Aggregate Concrete: Case Study. Applied Sciencesiences, Vol. 9, No. 617, DOI: https://doi.org/10.3390/app9040617.

Azarsa, P., and Gupta, R. (2017). Electrical Resistivity of Concrete for Durability Evaluation: A Review, Advances in Materials Science and Engineering, Vol. 2017, No. 8453095, DOI: https://doi.org/10.1155/2017/8453095.

Collepardi, M., Marcialis, A., and Turriziani, R. (1972). Penetration of chloride ions into cement pastes and concretes - collepardi, Journal of the American Ceramic Society, Vol. 55, No. 10, pp. 534-535.

COST 509 (1997). Corrosion and Protection of Metals in Contact with Concrete - Final Report.:

Delagrave, A., Marchand, J., Ollivier, J.-P., Julien, S., and Hazrati, K. (1997). Chloride binding capacity of various hydrated cement paste systems, Advanced Cement Based Materials, Vol. 6, No. 1, pp. 28-35, DOI: https://doi.org/10.1016/S1065-7355(97)90003-1 .

Faleschini, F., Zanini, M.A., and Hofer, L. (2018). Reliability-Based Analysis of Recycled Aggregate Concrete under Carbonation, Advances in Civil Engineering, Vol. 2018, p. 11.

Fernández Luco, L. (2001). "La durabilidad del hormigón: su relación con la estructura de poros y los mecanismos de transporte de fluidos." Durabilidad del Hormigón Estructural, Irassar, E.F. ed., AATH, Buenos Aires, p. 306.

Glass, G.K., Reddy, B., and Buenfeld, N.R. (2000). The participation of bound chloride in passive film breakdown on steel in concrete, Corrosion Science, Vol. 42, No. 11, pp. 2013-2021, DOI: https://doi.org/10.1016/S0010-938X(00)00040-8 .

Gómez, J.M., Agulló, L., and Vázquez, E. (2001). Cualidades Físicas y Mecánicas de los Agregados Reciclados de Concreto. Aplicación en Concretos, Concreto y Tecnología, Vol. 34, No. 5, pp. 799806. 
Hansen, T.C. (1986). Recycled aggregates and recycled aggregate concrete second state-of-the-art report developments 1945-1985, Matériaux et Constructions, Vol. 19, No. 111, pp. 201-246.

Huerta, E.O. (1997). Corrosión y Degradación de Materiales, Síntesis, Madrid, Spain.

Kim, H., and Bentz, D. (2008). Internal curing with crushed returned concrete aggregates for high performance concrete, NRMCA Concrete Technology Forum: Focus on ..., pp. 1-12.

Kovler, K., and Jensen, O.M. (2007). “General Concept and Terminology.” Internal Curing of Concrete - RILEM TC 196-ICC, State-of-the-Art Report, p. 5-13.

Leemann, A., and Loser, R. (2019). Carbonation resistance of recycled aggregate concrete, Construction and Building Materials, Vol. 204, pp. 335-341, DOI: https://doi.org/10.1016/j.conbuildmat.2019.01.162 .

Limbachiya, M.C., Leelawat, T., and Dhir, R.K. (2000). Use of recycled concrete aggregate in highstrength concrete Materials and Structures, Vol. 33, pp. 574-580.

Di Maio, A.A., Zega, C.J., and Traversa, L.P. (2005). Estimation of Compressive Strength of Recycled Concretes with the Ultras, onic Method: Journal of ASTM International, Vol. 2, No. 5, pp. 1-8, DOI: https://doi.org/10.1520/JAI12849 .

Martín-Pérez, B. (1999). Service Life Modelling of R. C. Highway Structures Exposed to Chlorides, University of Toronto, p. 168.

Monosi, S., Moriconi, G., Alveá, I., and Collepardi, M. (1989). Effect of water-cement ratio and curing time on chloride penetration into concrete, Materials Engineering, Vol. 1, No. 2, pp. 483-489.

Moro, J.M., Meneses, R.S., Ortega, N.F., and Aveldaño, R.R. (2016). "Corrosión de armaduras en elementos de hormigón reciclado con tratamientos de lechada de cemento." VII Congreso Internacional de la AATH, Salta, Argentina, p. 349-356.

Nilsson, L.-O. (1996). Interaction between microclimate and concrete - a prerequisite for deterioration, Construction and Building Materials, Vol. 10, No. 5, pp. 301-308, DOI: https://doi.org/10.1016/0950-0618(95)00046-1.

Omary, S., Ghorbel, E., Wardeh, G., and Nguyen, M.D. (2018). Mix Design and Recycled Aggregates Effects on the Concrete's Properties, International Journal of Civil Engineering, Vol. 16, No. 8, pp. 973-992, DOI: https://doi.org/10.1007/s40999-017-0247-y .

Ortega, N.F., Moro, J.M., Meneses, R.S., and Aveldaño, R.R. (2010). “Comportamiento Dinámico de Vigas de Hormigón Reciclado con sus Armaduras Corroídas." VI Congreso Internacional sobre Patología y Recuperación de Estructuras, Córdoba, Argentina, p. 12 p.

Otsuki, N., Miyazato, S., and Yodsudjai, W. (2003). Influence of Recycled Aggregate on Interfacial Transition Zone, Strength, Chloride Penetration and Carbonation of Concrete, Journal of Materials in Civil Engineering, Vol. 15, No. 5, pp. 443-451, DOI: https://doi.org/10.1061/(ASCE)08991561(2003)15:5(443).

Padmini, A.K., Ramamurthy, K., and Mathews, M.S. (2009). Influence of parent concrete on the properties of recycled aggregate concrete, Construction and Building Materials, Vol. 23, No. 2, pp. 829-836, DOI: https://doi.org/10.1016/J.CONBUILDMAT.2008.03.006 .

Rasheeduzzafar, and Khan, A. (1984). Recycled Concrete-A Source for New Aggregate, Cement, Concrete and Aggregates, Vol. 6, No. 1, pp. 17-27, DOI: https://doi.org/10.1520/CCA10349J .

Silva, R. V, Neves, R., De Brito, J., and Dhir, R.K. (2015). Carbonation behaviour of recycled aggregate concrete, Cement \& Concrete Composites, Vol. 62, pp. 22-32, DOI: https://doi.org/10.1016/j.cemconcomp.2015.04.017.

Sosa, M.E., Carrizo, L.E., Zega, C.J., and Villagrán, Y.A. (2018a). Water absorption of fine recycled aggregates: effective determination by a method based on elect. Conductivity, Materials and Structures, Vol. 51, p. 127, DOI: https://doi.org/10.1617/s11527-018-1248-2 .

Sosa, M.E., Villagrán Zaccardi, Y.A., Zega, C.J., and Di Maio, Á.A. (2018b). “Influence of Total and Effective Water-Cement Ratio on Compressive Strength of Concretes Made With Fine Recycled Concrete Aggregates. " IV International Conference Progress of Recycling in the Build Environment, 
Martins, I., Ulsen, C. and Villagrán, Y. eds., RILEM, Lisbon, p. 390-397.

Tanaka, K., Yada, K., Maruyama, I., Sato, R., and Kawai, K. (2004). "Study on corrosion of reinforcing bar in recycled concrete." International RILEM Conference on the Use of Recycled Materials in Building and Structures, p. 643-650.

Tang, L., and Nilsson, L.-O. (1993). Chloride binding capacity and binding isotherms of OPC pastes and mortars, Cement and Concrete Research, Vol. 23, No. 2, pp. 247-253.

Topçu, İ.B., and Şengel, S. (2004). Properties of concretes produced with waste concrete aggregate, Cement and Concrete Research, Vol. 34, No. 8, pp. 1307-1312, DOI: https://doi.org/10.1016/J.CEMCONRES.2003.12.019 .

Tuutti, K. (1982). Corrosion of steel in concrete, Swedish Cement and Concrete Institute, p. 468.

Villagrán-Zaccardi, Y.A., Zega, C.J., and Di Maio, Á.A. (2008). Chloride Penetration and Binding in Recycled Concrete, Journal of Materials in Civil Engineering, Vol. 20, No. 6, pp. 449-455, DOI: https://doi.org/10.1061/(ASCE)0899-1561(2008)20:6(449).

Villagran, Y., and Matiasich, C. (2004). Capacidad de fijación y adsorción de cloruros en cementos, Ciencia y Tecnología del Hormigón, No. 11, pp. 59-72.

Villagrán, Y.A.Z., Taus, V.L., Zega, C.J., Di Maio, A.A., and Traversa, L.P. (2005). "Propiedades de transporte en hormigones convencionales y reciclados y su influencia en la corrosiön de armaduras." Structural Concrete and Time - Proceedings of the fib Symposium.

Villagran Zaccardi, Y.A., and Di Maio, A.A. (2014). Electrical resistivity measurement of unsaturated concrete samples, Magazine of Concrete Research, Vol. 66, No. 10, pp. 484-491.

Villagrán Zaccardi, Y.A., Zega, C.J., and Di Maio, A.A. (2009). Hormigón con agregado reciclado proveniente de ambiente marino, ingreso de cloruro y corrosión de armaduras, Revista Hormigón, No. 47 , pp. 35-43.

Werle, A.P., Kulakowski, M.P., de Souza Kazmierczak, C., and Alcântara Alves Sentena, J. (2011). "Carbonation in Concrete with Recycled Concrete Aggregates." XII International Confrerence on Durability of Building Materials and Components, Porto, p. 8.

Whittington, H.W., McCarter, J., and Forde, M.C. (1981). The conduction of electricity through concrete, Magazine of Concrete Research, Vol. 114, No. 33, pp. 48-60.

Xiao, J. (2018). Recycled Aggregate Concrete Structures, Springer, Berlin.

Xiao, J., Lei, B., and Zhang, C. (2010). Effects of Recycled Coarse Aggregates on the Carbonation Evolution of Concrete, Key Engineering Materials, Vol. 417-418, pp. 697-700, DOI: https://doi.org/10.4028/www.scientific.net/KEM.417-418.697 .

Zega, C.J., Coelho Dos Santos, G.S., Pittori, A., and Di Maio, A.A. (2014). "Efecto del contenido de humedad del agregado grueso reciclado sobre la resistencia a compresión." VI Congreso Internacional de la AATH, p. 469-476.

Zega, C.J., Dos, G.S.C., Villagrán-zaccardi, Y.A., and Maio, A.A. Di (2016). Performance of recycled concretes exposed to sulphate soil for 10 years, CONSTRUCTION \& BUILDING MATERIALS, Vol. 102, pp. 714-721, DOI: https://doi.org/10.1016/j.conbuildmat.2015.11.025 .

Zega, C.J., Etcheverry, J.M., and Villagrán-Zaccar (2019). "NATURAL CARBONATION OF MULTIPLELY RECYCLED AGGREGATE CONCRETE." International Workshop CO2 Storage in Concrete, Djerbi, A., Omikrine-Metalssi, O. and Fen-Chong, T. eds., Paris, p. 117-123.

Zega, C.J., and Di Maio, Á.A. (2007). Efecto del agregado grueso reciclado sobre las propiedades del hormigón, Bol. Téc., IMME, Vol. 45, No. 2, pp. 1-11.

Zega, C.J., and Di Maio, A.A. (2003). "Influencia de las características de los agregados reciclados en la elaboración de hormigones." 15o Reunión Técnica AATH, AATH, Santa Fé (Argentina).

Zega, C.J., and Di Maio, Á.A. (2011). Use of recycled fine aggregate in concretes with durable requirements, Waste Management, Vol. 31, pp. 2336-2340, DOI: https://doi.org/10.1016/j.wasman.2011.06.011 .

Zega, C.J., Villagran-Zaccardi, Y.A., and Di Maio, A.A. (2015). "Chloride diffusion in recycled 
concretes made with different types of natural coarse aggregates." International Conference on Sustainable Structural Concrete, La Plata, p. 393-402.

Zega, C.J., Villagrán-Zaccardi, Y.A., and Di Maio, Á.A. (2010). Effect of natural coarse aggregate type on the physical and mechanical properties of recycled coarse aggregates, Materials and Structures, Vol. 43, pp. 195-202, DOI: https://doi.org/10.1617/s11527-009-9480-4 .

Zhao, Y., Dong, J., Wu, Y., Wang, H., Li, X., and Xu, Q. (2014). Steel corrosion and corrosion-induced cracking in recycled aggregate concrete, Corrosion Science, Vol. 85, pp. 241-250, DOI: https://doi.org/10.1016/j.corsci.2014.04.028 . 